PROCEEDINGS OF THE AMERICAN MATHEMATICAL SOCIETY

Volume 126, Number 7, July 1998, Pages 1973-1982

S 0002-9939(98)04699-1

\title{
A UNIFORM BOUNDEDNESS THEOREM FOR LOCALLY CONVEX CONES
}

\author{
WALTER ROTH
}

(Communicated by Palle E. T. Jorgensen)

\begin{abstract}
We prove a uniform boundedness theorem for families of linear operators on ordered cones. Using the concept of locally convex cones we introduce the notions of barreled cones and of weak cone-completeness. Our main result, though no straightforward generalization of the classical case, implies the Uniform Boundedness Theorem for Fréchet spaces.
\end{abstract}

\section{INTRODUCTION}

The theory of locally convex cones as developed in [2] deals with ordered cones that are not necessarily embeddable in vector spaces. A topological structure is introduced using order theoretical concepts. Staying close to the theory of locally convex spaces, this approach yields a rich duality theory including Hahn-Banach type extension and separation theorems for linear functionals (cf. [2], [5]). We shall review some of the main concepts. We globally refer to [2] for details and proofs:

An ordered cone (cf. [1] and [2]) is a set $P$ endowed with an addition $(a, b) \mapsto a+b$ and a scalar multiplication $(\alpha, a) \mapsto \alpha a$ for real numbers $\alpha \geq 0$. The addition is supposed to be associative and commutative, and there is a neutral element $0 \in P$. For the scalar multiplication the usual associative and distributive properties hold, i.e. $\alpha(\beta a)=(\alpha \beta) a,(\alpha+\beta) a=\alpha a+\beta a$ and $\alpha(a+b)=\alpha a+\beta a$ for all $a, b \in P$ and $\alpha, \beta \geq 0$. We have $1 a=a$ and $0 a=0$ for all $a \in P$. The cancellation law, stating that $a+c=b+c$ implies $a=b$, however, is not required in general. It holds if and only if the cone $P$ may be embedded into a real vector space. Also, $P$ carries a (partial) order, i.e. a reflexive transitive relation $\leq$ such that $a \leq b$ implies $a+c \leq b+c$ and $\alpha a \leq \alpha b$ for all $a, b, c \in P$ and $\alpha \geq 0$.

A full locally convex cone $(P, V)$ is an ordered cone $P$ that contains an abstract neighborhood system $V$, i.e. a subset of positive elements that is directed downward, closed for addition and multiplication by (strictly) positive scalars. The elements $v$ of $V$ define upper, resp. lower, neighborhoods for the elements of $P$ by

$$
v(a)=\{b \in P \mid b \leq a+v\}, \quad \text { resp. } a(v)=\{b \in P \mid a \leq b+v\},
$$

creating the upper, resp. lower, topologies on $P$. Their common refinement is called the symmetric topology. All elements of $P$ are supposed to be bounded below, i.e. for every $a \in P$ and $v \in V$ we have $0 \leq a+\rho v$ for some $\rho>0$.

Received by the editors July 15, 1996.

1991 Mathematics Subject Classification. Primary 46A08, 46A30.

Key words and phrases. Uniform boundedness theorem, locally convex cones.

(C)1998 American Mathematical Society 
Finally, a locally convex cone $(P, V)$ is a subcone of a full locally convex cone not necessarily containing the abstract neighborhood system $V$. Every locally convex ordered topological vector space is a locally convex cone in this sense, as it may be canonically embedded into a full locally convex cone (cf. [2], Example I.2.7).

For cones $P$ and $Q$ a mapping $T: P \rightarrow Q$ is called a linear operator if $T(a+b)=$ $T(a)+T(b)$ and $T(\alpha a)=\alpha T(a)$ holds for all $a, b \in P$ and $\alpha \geq 0$. If $(P, V)$ and $(Q, W)$ are locally convex cones, $T$ is called uniformly continuous (u-continuous) if for every $w \in W$ one can find $v \in V$ such that $T(a) \leq T(b)+w$ whenever $a \leq b+v$ for $a, b \in P$. Uniform continuity implies monotonicity in the following sense: If $a \leq b+v$ for all $v \in V$, then $T(a) \leq T(b)+w$ for all $w \in W$.

A linear functional on $P$ is a linear operator $\mu: P \rightarrow \overline{\mathbb{R}}=\mathbb{R} \cup\{+\infty\}$. In $\overline{\mathbb{R}}$ we consider the usual algebraic operations, in particular $\alpha+\infty=+\infty$ for all $\alpha \in \overline{\mathbb{R}}$, $\alpha \cdot(+\infty)=+\infty$ for all $\alpha>0$ and $0 \cdot(+\infty)=0$. Note that linear functionals can assume only finite values in elements $a \in P$ such that $-a \in P$ as well.

Endowed with the neighborhood system $W=\{\varepsilon \in \mathbb{R} \mid \varepsilon>0\}, \overline{\mathbb{R}}$ is a full locally convex cone. The u-continuous linear functionals from a locally convex cone $(P, V)$ to $\overline{\mathbb{R}}$ form again a cone, called the dual cone of $P$ and denoted by $P^{*}$. Continuity requires that a linear functional $\mu$ is monotone, and for a full cone $(P, V)$ it means just that $\mu(v) \leq 1$ holds for some $v \in V$ in addition.

The polar $v_{P}^{\circ}$ of a neighborhood $v \in V$ consists of all linear functionals $\mu$ on $P$ satisfying $\mu(a) \leq \mu(b)+1$, whenever $a \leq b+v$ for $a, b \in P$. The union of all polars yields $P^{*}$. We endow $P^{*}$ with the topology $w\left(P^{*}, P\right)$ of pointwise convergence of the elements of $P$, considered as functions on $P^{*}$ with values in $\overline{\mathbb{R}}$ with its usual topology. The polar $v_{P}^{\circ}$ of every neighborhood $v \in V$ is seen to be $w\left(P^{*}, P\right)$-compact and convex ([2], Theorem II.2.4).

A locally convex cone $(P, V)$ has the strict separation property if for all $a, b \in P$ and $v \in V$ such that $a \not \leq b+\rho v$ for some $\rho>1$ there is a linear functional $\mu \in v_{P}^{\circ}$ such that $\mu(a)>\mu(b)+1$ (cf. [2], II.2.12). Following Theorem 2.20 from [2], every locally convex cone with the strict separation property may be represented as a cone of $\overline{\mathbb{R}}$-valued functions on some set, or equivalently, as a cone of convex subsets of some locally convex ordered vector space.

Hahn-Banach type extension and separation theorems for locally convex cones were established in [2] and [5]. Theorem II.2.9 from [2] (a more general version is Theorem 4.1 from [5]) states that for a subcone $(Q, V)$ of $(P, V)$ every linear functional in $v_{Q}^{\circ}$ extends to an element of $v_{P}^{\circ}$.

In this paper we present a version of the classical Uniform Boundedness Theorem for locally convex cones. In Section 2 we introduce the notion of barreled locally convex cones. We show that completeness in a certain sense implies that a locally convex cone with a countable neighborhood base is barreled. Section 3 contains our main result which states that a family of uniformly continuous linear operators from a barreled locally convex cone to any locally convex cone with separation property satisfies a uniform boundedness principle.

\section{BARRELED CONES}

Throughout the following, let $(P, V)$ be a locally convex cone. Note that while all elements of $P$ are bounded below, they need not be bounded above. An element $a \in P$ is called bounded (above) (cf. [2], I.2.3) if for every $v \in V$ there is a $\lambda>0$ such that $a \leq \lambda v$. Bounded elements satisfy a modified version of the cancellation 
law (cf. [2], Lemma I.4.3), and continuous linear functionals take only finite values there.

A subset $A \subset P$ is said to be bounded if for every $v \in V$ there is a $\lambda>0$ such that $a \leq \lambda v$ and $0 \leq a+\lambda v$ for all $a \in A$. The elements of a bounded subset are therefore themselves bounded. We shall use a weaker notion of boundedness for subsets of $P$ in this paper, avoiding this requirement.

We shall say that a subset $A$ of $P$ is internally bounded if for every $v \in V$ there is a $\lambda>0$ such that $a \leq b+\lambda v$ for all $a, b \in A$.

A barrel is a convex subset $U$ of $P^{2}$ with the following properties:

(U1) For every $b \in P$ there is a $v \in V$ such that for every $a \in v(b) \cap(b) v$ there is a $\lambda>0$ such that $(a, b) \in \lambda U$.

(U2) For all $a, b \in P$ such that $(a, b) \notin U$ there is a $\mu \in P^{*}$ such that $\mu(c) \leq \mu(d)+1$ for all $(c, d) \in U$ and $\mu(a)>\mu(b)+1$.

2.1 Lemma. Let $(P, V)$ be a locally convex cone and $U \subset P^{2}$ a barrel.

(a) If $a \leq b$ for $a, b \in P$, then $(a, b) \in U$.

(b) If $(a+\varepsilon b,(1+\varepsilon) b) \in U$ for $a, b \in P$ and some $\varepsilon \geq 0$, then $(a, b) \in U$.

(c) If $(a, b) \in U$, if $a^{\prime}+c \leq a+d$ and $b^{\prime}+c \geq b+d$ for $a^{\prime}, b^{\prime}, c, d \in P$ and if $c$ is bounded, then $\left(a^{\prime}, b^{\prime}\right) \in U$.

Proof. (a) If $(a, b) \notin U$, by (U2) we could find some $\mu \in P^{*}$ such that $\mu(a)>$ $\mu(b)+1$. But this contradicts $\mu(a) \leq \mu(b)$, as all functionals in $P^{*}$ are monotone.

(b) If $(a, b) \notin U$, again using (U2) we could find $\mu \in P^{*}$ such that $\mu(a)>\mu(b)+1$ and $\mu(c) \leq \mu(d)+1$ for all $(c, d) \in U$. The latter implies that $\mu(a)+\varepsilon \mu(b) \leq$ $(1+\varepsilon) \mu(b)+1$ and contradicts the former, as $\mu(b)$ is finite. A similar argument involving (U2) shows statement (c).

If $(P, V)$ has the strict separation property, then every neighborhood $v \in V$ defines a barrel $U=\left\{(a, b) \in P^{2} \mid a \leq b+\rho v\right.$ for all $\left.\rho>1\right\}$. On the other hand, if $(P, V)$ is a full locally convex cone, then for every barrel $U$ and for $b=0$ there is by (U1) a neighborhood $v \in V$ such that $(v, 0) \in \lambda U$ for some $\lambda>0$. Let $a, b \in P$ such that $a \leq b+\lambda v$ and assume that $(a, b) \notin \lambda U$. Following (U2) there is a $\mu \in P^{*}$ such that $\mu(a)>\mu(b)+\lambda$ and $\mu(v) \leq \lambda$ as $(v / \lambda, 0) \in U$. But this contradicts $\mu(a) \leq \mu(b+v) \leq \mu(b)+\lambda$. We conclude that $\{(a, b) \mid a \leq b+v\} \subset \lambda U$.

In a locally convex vector space $E$ a barrel is defined to be an absolutely convex closed and absorbing subset $A$ of $E$ (cf. [6], II.7). The set $U=\left\{(a, b) \in E^{2} \mid\right.$ $a-b \in A\}$ then is seen to be a barrel in the sense of our definition. A locally convex vector space is said to be barreled if every barrel is a neighborhood of the origin. We adapt this definition to locally convex cones in the following way:

A locally convex cone $(P, V)$ is said to be barreled if for every barrel $U \subset P^{2}$ and every element $b \in P$ there is a neighborhood $v \in V$ and a $\lambda>0$ such that $(a, b) \in \lambda U$ for all $a \in v(b) \cap(b) v$.

2.2 Lemma. Let $(P, V)$ be a barreled locally convex cone. Then for every barrel $U \subset P^{2}$, every internally bounded subset $B \subset P$ and every $b \in B$ there is a neighborhood $v \in V$ and $\lambda>0$ such that $(a, b) \in \lambda U$ for all $a \in v\left(b^{\prime}\right) \cap\left(b^{\prime \prime}\right) v$ for some $b^{\prime}, b^{\prime \prime} \in B$.

Proof. For $U$ and $b \in B$ choose $v \in V$ and $\lambda>0$ as in the definition of a barreled cone. Let $\varepsilon>0$ be such that $b^{\prime} \leq b^{\prime \prime}+\varepsilon v$ for all $b^{\prime}, b^{\prime \prime} \in B$. Then $a \in v\left(b^{\prime}\right) \cap\left(b^{\prime \prime}\right) v$ implies that $a \leq b^{\prime}+v \leq b+(1+\varepsilon) v$. Adding $\varepsilon b$ on both sides yields $a+\varepsilon b \leq$ 
$(1+\varepsilon) b+(1+\varepsilon) v$ and $(a+\varepsilon b) /(1+\varepsilon) \leq b+v$. Similarly, using $a \in\left(b^{\prime \prime}\right) v$ one may check that $b \leq(a+\varepsilon b) /(1+\varepsilon)+v$. By our choice of $\lambda>0$ this shows $((a+\varepsilon b) /(1+\varepsilon), b) \in \lambda U$ and $(a+\varepsilon b,(1+\varepsilon) b) \in(1+\varepsilon) \lambda U$. Following Lemma 2.1(b), the latter implies $(a, b) \in(1+\varepsilon) \lambda U$, indeed.

Lemma 2.2 yields in particular that in a barreled locally convex cone, $B \times\{b\} \subset$ $\lambda U$ holds for every internally bounded set $B$ and $b \in B$ and every barrel $U$ with some $\lambda>0$.

In the theory of topological vector spaces Fréchet spaces are known to be barreled (cf. [6], II.7.1). In a similar vein we shall show that a modified notion of completeness will guarantee that a locally convex cone with a countable neighborhood base is barreled.

A locally convex cone $(P, V)$ is said to be weakly cone-complete if for all $b \in P$ and $v \in V$, every sequence $a_{n} \in v(b) \cap(b) v$ that converges to $b$ in the symmetric topology of $P$ and $\alpha_{n} \geq 0$ such that $\sum_{n=1}^{\infty} \alpha_{n}=1$, there is $a \in v(b) \cap(b) v$ such that

$$
\mu(a)=\sum_{n=1}^{\infty} \alpha_{n} \mu\left(a_{n}\right)
$$

for every $\mu \in P^{*}$ that is finite on $b$.

A neighborhood base for a locally convex cone $(P, V)$ is a subset $V_{0}$ of $V$ such that $v \geq v_{0}$ for all $v \in V$ and some $v_{0} \in V_{0}$.

2.3 Theorem. Every weakly cone-complete locally convex cone $(P, V)$ with a countable neighborhood base is barreled.

The proof of Theorem 2.3 will use the following technical result which generalizes Lemma 1.5 from [4] and is of some interest in itself. We give a slightly more general version than is actually required for our purposes.

2.4 Lemma. Let $P$ be an ordered cone, $A, B \subset P$ and $\Omega$ a set of monotone $\overline{\mathbb{R}}$ valued linear functionals on $P$ such that $\mu(b)<+\infty$ for all $b \in B$ and $\mu \in \Omega$. If for every $\rho>0$ there are $a \in A, b \in B$ and $\mu \in \Omega$ such that

$$
\mu(a)>\mu(b)+\rho,
$$

then at least one of the following statements holds:

(i) There are $a \in A$ and $b \in B$ such that for every $\rho>0$ there is $a \mu \in \Omega$ such that

$$
\mu(b)>\mu(a)+\rho .
$$

(ii) There is $a \mu \in \Omega$ such that for every $\rho>0$ there are $a \in A$ and $b \in B$ such that

$$
\mu(b)>\mu(a)+\rho .
$$

(iii) There are sequences $a_{i} \in A, b_{i} \in B, \mu_{i} \in \Omega$ and $\alpha_{i}, \beta_{i} \geq 0$ such that $\sum_{i=1}^{\infty} \alpha_{i}=\sum_{i=1}^{\infty} \beta_{i}=1$, satisfying the following: For every $\rho>0$ there are $a \in A, b \in B, \mu \in \Omega$ and $n_{0} \in \mathbb{N}$ such that

$$
\sum_{i=1}^{n} \alpha_{i} \mu\left(a_{i}\right)>\sum_{i=1}^{n} \alpha_{i} \mu\left(b_{i}\right)+\rho
$$


and

$$
\sum_{i=1}^{n} \beta_{i} \mu_{i}(a)>\sum_{i=1}^{n} \beta_{i} \mu_{i}(b)+\rho
$$

for all $n \geq n_{0}$.

Proof. Let us suppose that both statements (i) and (ii) fail, i.e. that

(i*) for all $a \in A$ and $b \in B$ there is a $\rho>0$ such that

$$
\mu(b) \leq \mu(a)+\rho \quad \text { for all } \mu \in \Omega,
$$

(ii*) for every $\mu \in \Omega$ there is a $\rho>0$ such that

$$
\mu(b) \leq \mu(a)+\rho \quad \text { for all } a \in A \text { and } b \in B .
$$

We shall show that under these conditions statement (iii) holds. We shall prove only the existence of the first series in our claim. The second one follows through an analogous construction. We set $\beta_{1}=1 / 2$ and select $a_{1} \in A, b_{1} \in B$ and $\mu_{1} \in \Omega$ such that

$$
\mu_{1}\left(a_{1}\right)>\mu_{1}\left(b_{1}\right)+2 .
$$

For $n \geq 2$ we choose $\beta_{n}, a_{n}, b_{n}$ and $\mu_{n}$ in the following way: Using (ii*) we find $0<\beta_{n} \leq 2^{-n}$ such that

$$
\beta_{n} \mu_{i}(b) \leq \beta_{n} \mu_{i}(a)+2^{-n}
$$

for all $a \in A, b \in B$ and $i=1, \ldots,(n-1)$. By (i*) there is a $\rho>0$ such that

$$
\sum_{i=1}^{n-1} \beta_{i} \mu\left(b_{i}\right) \leq \sum_{i=1}^{n-1} \beta_{i} \mu\left(a_{i}\right)+\rho
$$

for all $\mu \in \Omega$. Next we can find $a_{n} \in A, b_{n} \in B$ and $\mu_{n} \in \Omega$ such that

$$
\beta_{n} \mu_{n}\left(b_{n}\right)+(n+\rho)<\beta_{n} \mu_{n}\left(a_{n}\right) .
$$

Now adding the last two inequalities (and choosing $\mu=\mu_{n}$ in the first one) and using our assumption that $\mu(b)<+\infty$ for all $b \in B$ and $\mu \in \Omega$, we obtain

$$
n+\sum_{i=1}^{n} \beta_{i} \mu_{n}\left(b_{i}\right)<\sum_{i=1}^{n} \beta_{i} \mu_{n}\left(a_{i}\right) .
$$

Finally, we set $\beta=\sum_{n=1}^{\infty} \beta_{i} \leq 1$ and $\alpha_{i}=\beta_{i} / \beta$. We compute for every $m \geq n \in \mathbb{N}$ :

$$
\begin{aligned}
\sum_{i=1}^{m} \alpha_{i} \mu_{n}\left(a_{i}\right) & =(1 / \beta)\left(\sum_{i=1}^{n} \beta_{i} \mu_{n}\left(a_{i}\right)+\sum_{i=n+1}^{m} \beta_{i} \mu_{n}\left(a_{i}\right)\right) \\
& >(1 / \beta)\left(\sum_{i=1}^{n} \beta_{i} \mu_{n}\left(b_{i}\right)+n+\sum_{i=n+1}^{m}\left(\beta_{i} \mu_{n}\left(b_{i}\right)-2^{-i}\right)\right) \\
& >(1 / \beta)\left(\sum_{i=1}^{m} \beta_{i} \mu_{n}\left(b_{i}\right)+(n-1)\right) \\
& =\sum_{i=1}^{m} \alpha_{i} \mu_{n}\left(b_{i}\right)+(n-1) / \beta .
\end{aligned}
$$


The first part of statement (iii) is therefore evident. (We choose $n_{0} \geq \beta \rho+1$ and $\mu=\mu_{n_{0}}$.) For the second part one may use a similar argument, interchanging the respective parts of the sequences $\left(a_{n}\right)_{n \in \mathbb{N}},\left(b_{n}\right)_{n \in \mathbb{N}}$ and $\left(\mu_{n}\right)_{n \in \mathbb{N}}$.

We shall proceed now with the proof of Theorem 2.3: Let the locally convex cone $(P, V)$ be weakly cone-complete, and let $V_{0}=\left\{v_{n} \mid n \in \mathbb{N}\right\}$ be a neighborhood base for $(P, V)$ such that $v_{n} \geq v_{n+1}$ for all $n \in \mathbb{N}$. Let $U \subset P^{2}$ be a barrel and let $b \in P$. We may assume that condition (U1) holds for $U$ and $b$ with the neighborhood $v_{1}$.

Let us assume to the contrary of our claim that for every neighborhood $v_{n}$ there is $a_{n} \in v_{n}(b) \cap(b) v_{n}$ such that $\left(a_{n}, b\right) \notin n U$. By (U2) we can choose linear functionals $\mu_{n} \in P^{*}$ such that

$$
\mu_{n}(c) \leq \mu_{n}(d)+1 \quad \text { for all }(c, d) \in B \quad \text { and } \quad \mu_{n}\left(a_{n}\right)>\mu_{n}(b)+n
$$

for all $n \in \mathbb{N}$. This shows in particular that $\mu_{n}(b)$ is finite. Now we apply Lemma 2.4 to the sets $A=\left\{a_{n}\right\}, B=\{b\} \subset P$ and $\Omega=\left\{\mu_{n}\right\} \subset P^{*}$. Thus, at least one of the statements (i), (ii) or (iii) must hold.

For a fixed element $a \in A$ we have $(b, a) \in \lambda U$ for some $\lambda>0$ by (U1), hence $\mu(b) \leq \mu(a)+\lambda$ for all $\mu \in \Omega$. Statement (i) therefore cannot hold.

On the other hand, for any fixed $\mu \in \Omega$ there is an $n_{0}$ such that $\mu \in\left(v_{n_{0}}\right)_{P}^{\circ}$. Then from $b \leq a_{n}+v_{n}$ for all $n \in \mathbb{N}$ we infer that $\mu(b) \leq \mu\left(a_{n}\right)+1$ for all $n \geq n_{0}$. Thus statement (ii) can also not hold.

We may now look at the first series in statement (iii). There are sequences $a_{i} \in A, b_{i}=b \in B$ and $\alpha_{i} \geq 0$ with the stated properties. As $\mu(b)<+\infty$ for all $\mu \in \Omega$, we have $\mu(b)=\sum_{i=1}^{\infty} \alpha_{i} \mu(b)<+\infty$. From our construction, the sequence $\left(a_{i}\right)_{i \in \mathbb{N}}$ converges to $b$ in the symmetric topology of $P$ and $a_{n} \in v_{1}(b) \cap(b) v_{1}$ for all $n \in \mathbb{N}$. From the weak cone-completeness of $P$ we may therefore conclude that there is an $a \in v_{1}(b) \cap(b) v_{1}$ such $\mu(a)=\sum_{i=1}^{\infty} \alpha_{i} \mu\left(a_{i}\right)$ for every $\mu \in P^{*}$ such that $\mu(b)$ is finite. But this holds true for all our previously selected functionals $\mu \in \Omega$. We may now let $n$ tend to $+\infty$ in the first series of statement (iii). Thus for every $\rho>0$ there is a $\mu \in \Omega$ such that

$$
\mu(a)>\mu(b)+\rho .
$$

But this contradicts $(a, b) \in \lambda U$ for some $\lambda>0$, which follows from (U1) and implies that

$$
\mu(a) \leq \mu(b)+\lambda \quad \text { for all } \mu \in \Omega .
$$

2.5 Example. Let $(E,\|\|)$ be a Banach space, and let $P$ be the cone of all nonempty closed (resp. closed and bounded, resp. compact) convex subsets of $E$, endowed with the usual multiplication of sets by non-negative scalars, a slightly modified addition $\oplus$, where $A \oplus B$ denotes the closure of the usual sum of the sets $A$ and $B$, and the set inclusion as order. The neighborhood system $V$ is given by the positive multiples of the closed unit ball $\mathbb{B}$ in $E$. Thus $(P, V)$ is a locally convex cone.

We shall show that $(P, V)$ is weakly cone-complete: Let $\left(A_{n}\right)_{n \in \mathbb{N}}$ be a sequence in $P$ that converges to $B \in P$ with respect to the symmetric topology and satisfies $A_{n} \subset B \oplus \mathbb{B}$ and $B \subset A_{n} \oplus \mathbb{B}$ for all $n \in \mathbb{N}$. For a convergent series $\sum_{n=1}^{\infty} \alpha_{n}=1$ in $\mathbb{R}$ such that $\alpha_{n} \geq 0$ we set for $A$ the closure of the set

$$
\left\{\sum_{n=1}^{\infty} \alpha_{n} a_{n} \mid a_{n} \in A_{n} \quad \text { and }\left(a_{n}\right)_{n \in \mathbb{N}} \text { is bounded in } E\right\} .
$$


Note first that $A$ is not empty, as there is a $\rho>0$ such that $B \cap \rho \mathbb{B} \neq \emptyset$, hence $A_{n} \cap+(1+\rho) \mathbb{B} \neq \emptyset$ for all $n \in \mathbb{N}$ by our assumption. Consequently we may select at least one such bounded sequence $b_{n} \in A_{n} \cap(1+\rho) \mathbb{B}$, and we have $\sum_{n=1}^{\infty} \alpha_{n} b_{n} \in A$. It is easily verified that the set $A$ is convex (and resp. bounded, resp. compact), thus an element of $P$. Likewise, we check that $A \subset B \oplus \mathbb{B}$ and $B \subset A \oplus \mathbb{B}$. For a linear functional $\mu \in P^{*}$ there is (by the definition of $P^{*}$ ) a constant $\lambda>0$ such that $\mu(C) \leq \mu(D)+\lambda$, whenever $C \subset D \oplus \mathbb{B}$ for $C, D \in P$. For a fixed $n \in \mathbb{N}$, using the special sequence $\left(b_{n}\right)_{n \in \mathbb{N}}$ from above, we observe the following: If we choose $a_{i} \in A_{i}$ for $i=1, \ldots, n$ and set $a_{i}=b_{i}$ for $i>n$, then $\sum_{i=1}^{n} \alpha_{i} a_{i}=$ $\sum_{i=1}^{\infty} \alpha_{n} a_{n}-\sum_{i=n+1}^{\infty} \alpha_{n} b_{n}$. This shows

$$
\sum_{i=1}^{n} \alpha_{i} A_{i} \subset A \oplus\left\{-\sum_{i=n+1}^{\infty} \alpha_{i} b_{i}\right\} \subset A \oplus\left(\sum_{i=n+1}^{\infty} \alpha_{i}\right)(1+\rho) \mathbb{B},
$$

hence

$$
\sum_{i=1}^{n} \alpha_{i} \mu\left(A_{i}\right) \leq \mu(A)+\left(\sum_{i=n+1}^{\infty} \alpha_{i}\right)(1+\rho) .
$$

On the other hand we have

$$
A \subset \sum_{i=1}^{n} \alpha_{i} A_{i} \oplus\left(\sum_{i=n+1}^{\infty} \alpha_{i}\right)(B \oplus \mathbb{B}) .
$$

This shows

$$
\mu(A) \leq \sum_{i=1}^{n} \alpha_{i} \mu\left(A_{i}\right)+\left(\sum_{i=n+1}^{\infty} \alpha_{i}\right)(\mu(B)+\lambda) .
$$

If $\mu(B)$ is finite, we conclude that

$$
\mu(A)=\sum_{i=1}^{\infty} \alpha_{i} \mu\left(A_{i}\right)
$$

indeed.

Lemma 2.4 also provides the means to characterize internally bounded subsets of a locally convex cone through boundedness properties of elements of the dual cone.

2.6 Proposition. Let $(P, V)$ be a locally convex cone with the strict separation property. A subset $B \subset P$ is internally bounded if and only if for every neighborhood $v \in V$ and every convergent (with respect to the topology $w\left(P^{*}, P\right)$ ) net $\left(\mu_{\imath}\right)_{\imath \in \mathcal{I}}$ in $v_{P}^{\circ}$, there is a $\rho>0$ such that for all $a, b$ there is an $\imath_{0} \in \mathcal{I}$ and

$$
\mu_{\imath}(a) \leq \mu_{\imath}(b)+\rho \quad \text { for all } \imath \geq \imath_{0} .
$$

Proof. If $B \subset P$ is internally bounded, then for $v \in V$ there is a $\rho>0$ such that $a \leq b+\rho v$ for all $a, b \in B$, i.e.

$$
\mu(a) \leq \mu(b)+\rho
$$

for all $a, b \in B$ and $\mu \in v_{P}^{\circ}$. Thus our condition holds.

Now on the other hand let us assume that $B \subset P$ is not internally bounded, but that our condition holds anyway. We shall use Lemma 2.4 to obtain a contradiction: There are $v \in V$ and sequences $a_{n}, b_{n} \in B$ such that $a_{n} \not \leq b_{n}+n v$. The strict 
separation property for $(P, V)$ guarantees the existence of linear functionals $\mu_{n} \in v_{P}^{\circ}$ such that

$$
\mu_{n}\left(a_{n}\right)>\mu_{n}\left(b_{n}\right)+n .
$$

This implies in particular that $\mu_{n}\left(b_{n}\right)$ is finite, and therefore $\mu_{n}$ is even bounded as a function on $B$ by our condition. We set $A=B \subset P$ and $\Omega=\left\{\mu \in v_{P}^{\circ} \mid \mu(b)<\right.$ $+\infty$ for all $b \in B\}$ and apply Lemma 2.4. We check statements (i), (ii) and (iii) of the Lemma:

(i) Assume that for $a, b \in B$ there are $\mu_{n} \in v_{P}^{\circ}$ such that $\mu_{n}(a)>\mu_{n}(b)+n$. Using the $w\left(P^{*}, P\right)$-compactness of $v_{P}^{\circ}$ we find a convergent subnet $\left(\mu_{\imath}\right)_{\imath \in I}$ of $\left(\mu_{n}\right)_{\in \mathbb{N}}$. But this contradicts our condition. So does statement (ii), quite obviously.

(iii) We select sequences $\mu_{i} \in \Omega$ and $\beta_{i} \geq 0$ and discuss the second series in statement (iii). The sequence $\nu_{n}=\sum_{i=1}^{n} \beta_{i} \mu_{i} \in v_{P}^{\circ}$ is convergent in $v_{P}^{\circ}$, as

$$
\mu(a)=\sum_{i=1}^{\infty} \beta_{i} \mu_{i}(a) \quad \text { for all } a \in P
$$

defines a linear functional in $v_{P}^{\circ}$. (Convergence of this series in $\overline{\mathbb{R}}$ for each $a \in P$ results from the fact that $0 \leq a+\lambda v$ for some $\lambda>0$, hence $\mu_{i}(a) \geq-\lambda$ for all $i \in \mathbb{N}$.) Statement (iii) of Lemma 2.4 therefore contradicts our condition as well.

2.7 Corollary. Let $(P, V)$ be a locally convex cone with the strict separation property. A subset $B \subset P$ is bounded if and only if every linear functional $\mu \in P^{*}$ is bounded as a function on $B$.

Proof. Note that a set $B \subset P$ is bounded if and only if $B_{0}=B \cup\{0\}$ is internally bounded. Let $v \in V$. The condition in Proposition 2.6 for $B_{0}$ yields immediately that every $\mu \in v_{P}^{\circ}$ is bounded on $B$. If on the other hand every linear functional $\mu \in v_{P}^{\circ}$ is bounded on $B$, and if the net $\mu_{\imath} \in v_{P}^{\circ}$ converges to $\mu \in v_{P}^{\circ}$, then $\mu(a) \leq \mu(b)+\rho$ for all $a, b \in P$ implies the condition in 2.6.

\section{A Uniform Boundedness Theorem}

We are now ready to formulate our main result. It implies the classical Uniform Boundedness Theorem for locally convex vector spaces as a special case.

3.1 Theorem. Let $(P, V)$ and $(Q, W)$ be locally convex cones, and let $\mathcal{T}$ be a family of u-continuous linear operators from $P$ to $Q$. Suppose that for every $b \in P$ and $w \in W$ there is a $v \in V$ such that for every $a \in v(b) \cap(b) v$ there is $a \lambda>0$ such that

$$
T(a) \leq T(b)+\lambda w \quad \text { for all } T \in \mathcal{T} .
$$

If $(P, V)$ is barreled and $(Q, W)$ has the strict separation property, then for every internally bounded set $B \subset P$, every $b \in B$ and $w \in W$ there is $a v \in V$ and $a$ $\lambda>0$ such that

$$
T(a) \leq T(b)+\lambda w \quad \text { for all } T \in \mathcal{T}
$$

and all $a \in v\left(b^{\prime}\right) \cap\left(b^{\prime \prime}\right) v$ for some $b^{\prime}, b^{\prime \prime} \in B$. 
Proof. Let $w \in W$ and set

$$
U=\left\{(a, b) \in P^{2} \mid T(a) \leq T(b)+\rho w \quad \text { for all } \rho>1 \text { and } T \in \mathcal{T}\right\} .
$$

We shall show that $U$ is a barrel for $P$ :

(U1) For $b \in P$ choose $v \in V$ as in the assumption of the theorem. Then for every $a \in v(b) \cap(b) v$ there is a $\lambda>0$ such that $T(a) \leq T(b)+\lambda w$ for all $T \in \mathcal{T}$, i.e. $(a, b) \in \lambda U$.

(U2) For all $a, b \in P$ such that $(a, b) \notin U$ there is a $T \in \mathcal{T}$ such that $T(a) \not \leq$ $T(b)+\rho w$ for some $\rho>1$. Because $Q$ has the strict separation property, there is a $\nu \in w_{Q}^{\circ}$ such that $\nu(T(a))>\nu(T(b))+1$. Set $\mu=T^{*}(\nu) \in P^{*}$. (By the definition of the adjoint of a u-continuous linear operator (cf. [2], II.2.15) we have $\mu(a)=T^{*}(\nu)(a)=\nu(T(a))$ for all $a \in P$.) The functional $\mu$ fulfils the requirement of (U2).

Thus $U$ is a barrel, indeed, and by Lemma 2.2 then for every internally bounded set $B \subset P$ and $b \in B$ there is a neighborhood $v \in V$ and a $\lambda>0$ such that $(a, b) \in \lambda U$ for all $a \in v\left(b^{\prime}\right) \cap\left(b^{\prime \prime}\right) v$ for some $b^{\prime}, b^{\prime \prime} \in B$. By the definition of $U$ this proves

$$
T(a) \leq T(b)+(\lambda+1) w \quad \text { for all } T \in \mathcal{T} .
$$

Using Lemma 2.1 one might expand the range of the statement of Theorem 3.1: The consequence of the Theorem still holds true for all elements $a^{\prime}, b^{\prime} \in P$ such that $a^{\prime}+c \leq a+d$ and $b^{\prime}+c \geq b+d$ for some $c, d \in P$ such that $c$ is bounded, and $a \in v\left(b^{\prime}\right) \cap\left(b^{\prime \prime}\right) v$ for some $b^{\prime}, b^{\prime \prime} \in B$.

We shall illustrate the statement of Theorem 3.1 with a simple example:

3.2 Example. Let $P$ be the cone of all lower semicontinuous $\overline{\mathbb{R}}$-valued functions $f$ on the interval $[0,1]$ such that $f(x)=+\infty$ for all $x$ in a neighborhood of 1 , endowed with the pointwise algebraic operations and order and the neighborhood system $V=\{\varepsilon \in \mathbb{R} \mid \varepsilon>0\}$. Thus $(P, V)$ is a locally convex cone, but not a full cone, as the constant functions are not contained in $P$.

For weak cone-completeness, let $\left(f_{n}\right)_{n \in \mathbb{N}}$ be a sequence in $P$ that converges to the function $g \in B$ with respect to the symmetric topology and satisfies $f_{n} \leq$ $g+1$ and $g \leq f_{n}+1$ for all $n \in \mathbb{N}$. We infer that there is a $\rho>0$ such that $0 \leq f_{n}+\rho$ for all $n \in \mathbb{N}$. For a series $\sum_{n=1}^{\infty} \alpha_{n}=1$ in $\mathbb{R}$ such that $\alpha_{n} \geq 0$ we set $f(x)=\sum_{n=1}^{\infty} \alpha_{n} f_{n}(x)$. This series converges in $\overline{\mathbb{R}}$ for every $x \in[0,1]$, and $f$ is lower semicontinuous. Moreover, $f \leq g+1$ and $g \leq f+1$. For every linear functional $\mu \in P^{*}$ there is (by the definition of $P^{*}$ ) a constant $\lambda>0$ such that $\mu(h) \leq \mu(l)+\lambda$, whenever $h \leq l+1$ for functions $h, l \in P$. We observe for a fixed $n \in \mathbb{N}:$ As

$$
\sum_{i=1}^{n} \alpha_{i} f_{i} \leq f+\rho \sum_{i=n+1}^{\infty} \alpha_{i}
$$

holds, we infer that

$$
\sum_{i=1}^{n} \alpha_{i} \mu\left(f_{i}\right) \leq \mu(f)+\lambda \rho \sum_{i=n+1}^{\infty} \alpha_{i}
$$


On the other hand,

$$
f \leq \sum_{i=1}^{n} \alpha_{i} f_{i}+\left(\sum_{i=n+1}^{\infty} \alpha_{i}\right)(g+1)
$$

shows that

$$
\mu(f) \leq \sum_{i=1}^{n} \alpha_{i} \mu\left(f_{i}\right)+\left(\sum_{i=n+1}^{\infty} \alpha_{i}\right)(\mu(g)+\lambda) .
$$

If $\mu(g)<+\infty$, this yields

$$
\mu(f)=\sum_{i=1}^{\infty} \alpha_{i} \mu\left(f_{i}\right)
$$

indeed.

Now for $n \in \mathbb{N}$ we set $t_{n}(x)=n x^{n}$ and define linear operators $T_{n}: P \rightarrow P$ by $T_{n}(f)=t_{n} f$. These operators are u-continuous, as $f \leq g+\varepsilon$ implies that $T_{n}(f) \leq T_{n}(g)+n \varepsilon$ for all $f, g \in P$. Let us check the statement of Theorem 3.1: For an internally bounded set $B \subset P$ there is $0<\delta<1$ such that $g(x)=+\infty$ for all $\delta \leq x \leq 1$ and all $g \in B$. We choose a function $g \in B$ and the neighborhood $w=1 \in V$. Then for every function $f \in P$ such that $f \leq g^{\prime}+1$ and $g^{\prime \prime} \leq f+1$ for some $g^{\prime}, g^{\prime \prime} \in B$ we realize that $f(x)=+\infty$ holds for all $\delta \leq x \leq 1$ with the same $0<\delta<1$ from above. Therefore

$$
T_{n}(f) \leq T_{n}(g)+n \delta^{n},
$$

and as $n \delta^{n} \rightarrow 0$ as $n \rightarrow \infty$, there is a $\lambda>0$ such that

$$
T_{n}(f) \leq T_{n}(g)+\lambda
$$

for all such functions $f \in P$ and $n \in N$. The constant $\lambda>0$ depends however on the set $B$ and may not be chosen independently.

\section{REFERENCES}

1. B. Fuchssteiner and W. Lusky, Convex cones, vol. 56, North Holland Math. Studies, 1981. MR 83m: 46018

2. K. Keimel, W. Roth, Ordered cones and approximation, Lecture Notes in Mathematics, vol. 1517, Springer Verlag, Heidelberg-Berlin-New York, 1992. MR 93i:46017

3. A.P. Robertson and W.J. Robertson, Topological vector spaces, Cambridge Tracts in Mathematics and Mathematical Physics, 1964. MR 28:5318

4. W. Roth, A combined approach to the Fundamental theorems for normed spaces, Bulletin Acad. Sinica 22 (1) (1994), 83-89. MR 95e:46019

5. W. Roth, Hahn-Banach type theorems for locally convex cones, to appear.

6. H.H. Schaefer, Topological vector spaces, Springer Verlag, Heidelberg-Berlin-New York, 1980. MR 49:7722 (1971 printing)

Department of Mathematics, Universiti Brunei Darussalam, Bandar Seri Begawan 2028, Brunei Darussalam

E-mail address: roth@ubd.edu.bn 